\title{
Cellulose nanofiber aerogel as a promising biomaterial for customized oral drug delivery
}

\author{
This article was published in the following Dove Press journal: \\ International Journal of Nanomedicine \\ 14 March 2017 \\ Number of times this article has been viewed
}

\author{
Jyoti Bhandari' \\ Harshita Mishra' \\ Pawan Kumar Mishra ${ }^{2}$ \\ Rupert Wimmer ${ }^{2,3}$ \\ Farhan J Ahmad' \\ Sushama Talegaonkar' \\ 'Department of Pharmaceutics, \\ Jamia Hamdard, New Delhi, India; \\ ${ }^{2}$ Department of Wood Science, \\ Mendel University in Brno, Brno, \\ Czech Republic; ${ }^{3}$ Institute for Natural \\ Materials Technology, Department \\ of Agrobiotechnology, IFA-Tulln, \\ University of Natural Resources and \\ Life Sciences, Vienna, Austria
}

\begin{abstract}
Cellulose nanofiber (CNF) aerogels with favorable floatability and mucoadhesive properties prepared by the freeze-drying method have been introduced as new possible carriers for oral controlled drug delivery system. Bendamustine hydrochloride is considered as the model drug. Drug loading was carried out by the physical adsorption method, and optimization of drug-loaded formulation was done using central composite design. A very lightweight-aerogelwith-matrix system was produced with drug loading of $18.98 \% \pm 1.57 \%$. The produced aerogel was characterized for morphology, tensile strength, swelling tendency in media with different $\mathrm{pH}$ values, floating behavior, mucoadhesive detachment force and drug release profiles under different $\mathrm{pH}$ conditions. The results showed that the type of matrix was porous and woven with excellent mechanical properties. The drug release was assessed by dialysis, which was fitted with suitable mathematical models. Approximately $69.205 \% \pm 2.5 \%$ of the drug was released in 24 hours in medium of $\mathrm{pH} 1.2$, whereas $\sim 78 \% \pm 2.28 \%$ of drug was released in medium of $\mathrm{pH} 7.4$, with floating behavior for $\sim 7.5$ hours. The results of in vivo study showed a 3.25 -fold increase in bioavailability. Thus, we concluded that CNF aerogels offer a great possibility for a gastroretentive drug delivery system with improved bioavailability.

Keywords: cellulose nanofiber, aerogel, controlled release, gastroretentive, floating behavior, swelling behavior, mucoadhesion, bioavailability
\end{abstract}

\section{Introduction}

Drug delivery systems, especially those having sustained and targeted release, have received more attention in the past few decades due to the potential benefits that they offer, such as decrease in side effects and enhancement in therapeutic efficacy for a prolonged period of time, with the possibility for dose reduction. ${ }^{1}$ Further, oral controlled drug delivery is always considered the ideal drug delivery system. Oral route presents benefits relative to the intravenous (iv) route in cancer therapy in several ways. Though the iv route leads to immediate and complete bioavailability as well as accurate dosing, this route could be hazardous because high concentration of the drug is delivered to normal tissues. Other advantages of the oral route include patient compliance, painless delivery, economical advantage and improvement in daily dosage regime of the patient. ${ }^{2}$ In order to enhance the gastric residence time of the oral formulations, different approaches have been studied, such as altered density formulations, floating systems, bioadhesive formulations, swelling systems, ion exchange resins, porous systems, osmoregulated formulations and effervescent formulations. ${ }^{3}$

Nanofibers (NFs) possess a huge potential in biomedicine as a carrier for controlled drug delivery because of their unique attributes, such as high surface area per unit mass, extensive porosity and strength with flexibility, in addition to being economical. ${ }^{4}$ Due to these significant physicochemical properties, NFs are exploited
Correspondence: Sushama Talegaonkar Nanoformulation Research Laboratory, Department of Pharmaceutics,

Faculty of Pharmacy, Jamia Hamdard, Mehrauli-Badarpur Road, Hamdard

Nagar, New Delhi I 10062, India

Tel+9l 98 I84535।8

Email stalegaonkar@gmail.com 
for a wide range of applications. In the field of biotechnology, NFs serve a variety of purposes, such as in drug delivery, dental applications, wound dressing medicinal implants and tissue engineering. ${ }^{5}$ They serve as carriers for the delivery of drugs such as antimicrobials, enzymes, other drugs, antioxidants, flavors and functional group compounds. Besides the medicinal purposes, they are also used in filtration media as biosensors, in military protective clothing and for various other industrial applications such as in the food industry. ${ }^{6}$ NFs can also be used for the controlled release of compounds by protecting and stabilizing the compound, being slowly released under suitable conditions. ${ }^{7}$ Cellulose, which is a relatively stable polymer with hydrogen bonding, serves as a good source of NFs. Cellulose nanofibers (CNFs) possess several advantages such as good flexibility, good elasticity, low density, low toxicity and a relatively reactive surface that can be used for grafting specific groups, in addition to being renewable and cheap. ${ }^{8}$

CNF can be broadly classified into two categories. One is obtained from plant sources and the next is from bacterial sources. The plant-sourced cellulose is further classified into wood- and nonwood-sourced fiber. The nonwood source includes agricultural crops and the agricultural by-products obtained from corn, wheat, rice, barley, sugarcane, pineapple, banana, coconut tree, potato pulp, sweet beet pulp and soy. Bacterial cellulose, also referred to as microbial cellulose, has gained particular attention recently, especially in biomedical and tissue engineering. Bacterial cellulose is mainly produced by Acetobacter xylinum, ${ }^{9}$ which is a Gram-negative strain of acetic acid-producing bacteria. However, other bacteria such as Acanthamoeba, Achromobacter, Zoogloea, Agrobacterium, Pseudomonas, Alcaligenes, Sarcina and Rhizobium also secrete cellulose fibers extracellularly. Microbial or bacterial cellulose (BC) is chemically identical to plant cellulose (PC), though they possess different macromolecular structures and physical properties. ${ }^{10}$

CNFs generally are converted into the aerogel form during drug adsorption and subsequent freeze-drying. Aerogel is another form of CNF, which is a foam-like structure with very light weight, is highly porous and can be prepared by freeze-drying technique. The main aim of our project was to explore the properties of the obtained CNF for use in oral drug delivery. Because of the hydrophilic nature of bendamustine hydrochloride and the ability of cellulose to swell in aqueous media, bendamustine hydrochloride was chosen as a model cancer drug.

\section{Materials and methods Materials}

CNF was procured from The Process Development Center, University of Maine, Orono, ME, USA. Bendamustine hydrochloride was obtained as a gift sample from Fresenius Kabi Oncology Limited (Gurgaon, Haryana, India). All the other chemicals used were of analytical grade.

\section{Preparation of plain CNF}

The procured CNFs were prepared at the US Forest Service's Cellulose Nanomaterials Pilot Plant at the Forest Products Laboratory (FPL, University of Maine). The source of the cellulose for the preparation of these NFs was wood pulp. As per the specifications provided, the material supplied was $98 \mathrm{wt} \%$ dry powder, with nominal fiber diameter of $50 \mathrm{~nm}$ and length of several microns. Surface of the fibers was hydrophilic, and Brunauer, Emmett and Teller (BET) surface area was $31-33 \mathrm{~m}^{2} / \mathrm{g}$.

\section{Preparation of drug-loaded CNF (DCNF) aerogels}

The method reported by Kolakovic et al ${ }^{11}$ was used with slight modification. Saturated solutions of the drug were prepared in phosphate buffer ( $\mathrm{pH}$ 2.5) maintaining the volume constant at $4 \mathrm{~mL}$. Different amounts of CNFs were added to it in order to get the ratios of CNF: drug as 1:0.5, 1:1, 1:2, 1:3, 1:4 and 1:5. The solution was magnetically stirred for $1-16$ hours for preliminary tests at 1,500 rpm. The prepared suspension was transferred to centrifuge tubes and was centrifuged at $14,000 \mathrm{rpm}$ for 15 minutes. The supernatant was decanted and its volume was measured. Absorbance of the supernatant solution after suitable dilution was measured at $233 \mathrm{~nm}$ using ultraviolet spectrophotometer. Concentration of the drug in the supernatant was then calculated. The drug loading was further optimized using the two-factor central composite design, as suggested by the Design-Expert ${ }^{\circledR}$ (Stat-Ease, Inc) software for design of experiments (DOE) for the one dependent attribute, namely, drug loading. The independent variables selected for the optimization were as follows: stirring time varied from 3 to 8 hours and the CNF ratio varied from 0.6 to 3 . The settled DCNFs were collected and lyophilized using mannitol (1\%) as the cryoprotectant. The primary drying was performed at $-30^{\circ} \mathrm{C}$ for 72 hours, followed by the secondary drying, wherein the temperature was first increased to $-10^{\circ} \mathrm{C}$ for 4 hours and then to $-5^{\circ} \mathrm{C}$ for 18 hours and $0^{\circ} \mathrm{C}$ for the next 2 hours. Pressure was maintained at 150-225 mTor (20-30 Pa) according to Valo et al. ${ }^{12}$ 


\section{Characterization of DCNF aerogel system}

The aerogels were characterized on the basis of various parameters, such as surface morphology (determined using scanning electron microscopy [SEM] and transmission electron microscopy [TEM]), swelling indexes in media with different $\mathrm{pH}$ values, floating behavior, tensile strength, mucoadhesion, drug content and in vitro release in media with different $\mathrm{pH}$ values.

\section{Determination of drug loading and drug entrapment efficiency}

Drug was loaded onto the particles by physical adsorption. Drug content of the CNFs was calculated by an indirect method, ${ }^{13}$ whereby the supernatant collected was analyzed for the amount of drug present in it, and finally the drug loading was calculated using the following formula:

$$
\text { Drug loading }(\mathrm{DL} \%)=\frac{D_{\mathrm{i}}-D_{\mathrm{f}}}{C} \times 100
$$

where $D_{\mathrm{i}}$ and $D_{\mathrm{f}}$ represent the initial amount of drug added and the measured amount of free drug in the supernatant, respectively. $C$ is the total amount of NFs recovered.

For the optimized drug-loaded formulation, the entrapment efficiency was calculated by the following formula:

$$
\text { Entrapment efficiency }(\mathrm{EE} \%)=\frac{D_{\mathrm{i}}-D_{\mathrm{f}}}{D_{\mathrm{i}}} \times 100
$$

\section{SEM study}

The surface morphology of the aerogel was examined by SEM. The aerogel was mounted on aluminum stubs using double-sided carbon adhesive tape and sputter-coated with conductive gold-palladium. They were viewed with an EVO LS 10 (Carl Zeiss, Brighton, Germany) scanning electron microscope operating at an accelerating voltage of $13.52 \mathrm{kV}$ under high vacuum. Data analysis was done using Smart SEM software program.

\section{TEM study}

TEM study was carried out for the provided CNF and the optimized DCNF. The samples were mounted on copper grids with the salt of a heavy metal, viz, $1 \%$ uranyl acetate, for negative staining, followed by sample drying. They were then analyzed by TEM at an accelerating voltage of $100 \mathrm{kV}$; data acquisition was done on the AMT Image Capture Engine.

\section{Swelling index}

In order to evaluate the swelling behavior, $\mathrm{CNF}$ and DCNF aerogel samples of known weights were placed in test tubes containing $10 \mathrm{~mL}$ of $\mathrm{HCl}$ buffer $\mathrm{pH} 1.2$ and phosphate buffer pH 7.4 for 24 and 48 hours at $37^{\circ} \mathrm{C}$. Thereafter, samples were collected back and the water on the surfaces of the samples was removed with filter paper. Samples were then weighed in the wet condition. ${ }^{14}$ The swelling ratio for each sample was calculated according to the following equation:

$$
\text { Swelling ratio }(\%)=\frac{M_{\mathrm{w}}-M_{\mathrm{d}}}{M_{\mathrm{d}}} \times 100
$$

where $M_{\mathrm{d}}$ is the initial weight of the drug-loaded NFs and $M_{\mathrm{w}}$ is the final weight of the drug-loaded NFs in wet condition.

\section{Mucoadhesion test}

Mucoadhesive strength was determined with the help of a texture analyzer (TA.XTplus; Stable Micro Systems, Godalming, Surrey, UK). The aerogel $\left(\sim 2 \mathrm{~cm}^{2}\right)$ was attached to the cylindrical probe using double-sided adhesive tape. Goat stomach mucosa $(\sim 20 \times 20 \mathrm{~mm})$ was hydrated for 15 minutes at $37^{\circ} \mathrm{C} \pm 0.5^{\circ} \mathrm{C}$ and then placed on the holder stage of the mucoadhesive holder. ${ }^{15}$ The instrument was run at a load of $200 \mathrm{~g}$, and the weight required to detach the fiber from the mucosa was determined.

\section{Tensile strength}

Tensile strength of the CNF and DCNF aerogel formulations was determined using a texture analyzer (TA.XTplus). The aerogel was cut into pieces of dimensions $6 \times 2 \mathrm{~cm}^{2}$ with thickness of $\sim 1 \mathrm{~mm}$ and was placed in a vertical position along the axis of the texture analyzer, using clamps. The instrument was run at a load of $3 \mathrm{~g} / \mathrm{cm}^{2}$ after necessary adjustments. The force required to break the formulation was determined. ${ }^{16}$

\section{Floating time}

In order to calculate the floating time, DCNF aerogel with dimensions of $\sim 3 \times 3 \mathrm{~cm}^{2}$ was taken and placed into $\mathrm{HCl}$ buffer of $\mathrm{pH} 1.2$ and maintained at $37^{\circ} \mathrm{C}$. Total floating time, ie, the time period for which the formulation remained buoyant, was measured. ${ }^{17}$

\section{In vitro drug release}

DCNF aerogels were evaluated for in vitro drug release by using a dialysis membrane. $\mathrm{HCl}$ buffer $(\mathrm{pH} 1.2)$ was used 
as the release medium in the studies as the formulation is intended to release the drug in the stomach. The dialysis bags (molecular weight cutoff: $8-10 \mathrm{kDa}$; Spectra/Por ${ }^{\circledR}$; Spectrum Laboratories, Inc, Los Angeles, CA, USA) were soaked in double-distilled water for 12 hours before use. Pure bendamustine solution and its equivalent DCNF were placed in separate dialysis bags. The bags were suspended in $200 \mathrm{~mL}$ of receiving phase, ie, $\mathrm{HCl}$ buffer ( $\mathrm{pH} 1.2$ ), and placed in an incubator shaker maintained at $37^{\circ} \mathrm{C}$ and $100 \mathrm{rpm}$. Aliquots were withdrawn at various time points. Sink condition was maintained throughout the experiment. The concentration of bendamustine hydrochloride in the aliquots was analyzed by ultraviolet spectrophotometry at $233 \mathrm{~nm}$, and the cumulative percentage of drug release was determined. In order to compare the differences in the drug release profiles obtained from the CNF films in media of different $\mathrm{pH}$ values, the same study was carried out in phosphate buffer, $\mathrm{pH} 7.4$. The results obtained from the media with two different $\mathrm{pH}$ values were compared.

\section{Drug release kinetics}

The kinetics of bendamustine hydrochloride release from the DCNF in media of $\mathrm{pH} 1.2$ was studied using different kinetic models, including zero-order release, first-order release, Higuchi and Korsmeyer-Peppas models.

\section{In vivo studies}

\section{HPLC analysis in plasma}

For the HPLC analysis, the method reported by Xie et $\mathrm{al}^{18}$ was used, with slight modification. Separation was carried out in isocratic mode with TC-C18 column $(4.6 \times 250 \mathrm{~mm}$, $5 \mu \mathrm{m}$ inner diameter; Agilent, Waldbronn, Germany). The mobile phase consisted of acetonitrile (ACN): $10 \mathrm{mM}$ potassium dihydrogen phosphate solution in the ratio 32:68 (v/v, $\mathrm{pH} 2.5)$. The flow rate was $1.0 \mathrm{~mL} \cdot \mathrm{min}^{-1}$; column temperature was $30^{\circ} \mathrm{C}$; and ultraviolet detector was set at $231 \mathrm{~nm}$. The calibration curve in plasma was prepared for different concentrations ranging from 250 to $5,000 \mathrm{ng} \cdot \mathrm{mL}^{-1}$. Dilution was carried out using the mobile phase. For preparation of sample, precipitation of the proteins in the plasma was carried out using 10\% perchloric acid-methanol solution, which was then centrifuged at 15,000 rpm for 15 minutes. Subsequently, $20 \mu \mathrm{L}$ of supernatant was withdrawn and injected into the chromatographic system.

\section{Animals}

The animal study protocol to carry out in vivo studies was duly approved by the Jamia Hamdard Institutional Animal
Ethics Committee (approval number 1192, 2015). Guidelines of the Committee for the Purpose of Control and Supervision of Experiments on Animals (CPCSEA) were followed during the experiments. Wistar rats (200-300 g) of either sex were maintained under standard laboratory conditions (temperature $25^{\circ} \mathrm{C} \pm 2{ }^{\circ} \mathrm{C}$ and relative humidity $55 \% \pm 5 \%$ ). They were housed in polypropylene cages (two per cage) with free access to standard laboratory diet (Lipton Feed, Mumbai, Maharashtra, India) and water ad libitum.

\section{Method}

For the in vivo pharmacokinetic study, the animals were divided into three groups. Group A was given marketed preparation (Bendit) intravenously via the tail vein keeping the volume $0.5 \mathrm{~mL}$, and Groups B and C were respectively given pure drug solution and DCNF formulation orally by using a feeding tube. The dose calculation of the rats was based on body weight and was determined to be $27.75 \mathrm{mg} / \mathrm{kg}$.

Human equivalent dose of bendamustine hydrochloride is $4.2-5.5 \mathrm{mg} / \mathrm{kg} .{ }^{19}$ Accordingly, rat dose was calculated as follows:

$$
\begin{aligned}
& \text { Rat dose }(\mathrm{mg} / \mathrm{kg}) \\
& =\text { Human equivalent dose }(\mathrm{mg} / \mathrm{kg}) \times \frac{\text { Human } \mathrm{Km}}{\text { Rat } \mathrm{Km}} \\
& =4.5 \times 37 / 6 \\
& =27.75 \mathrm{mg} / \mathrm{kg}
\end{aligned}
$$

Note that $\mathrm{Km}$ is body weight $(\mathrm{kg}$ ) divided by BSA (body surface area, $\mathrm{m}^{2}$ ). Average $\mathrm{Km}$ for human is 37 ; average $\mathrm{Km}$ for rat is 6 . At the time of blood sampling, each animal was anesthetized with diethyl ether, and blood samples $(1 \mathrm{~mL})$ were collected from the retroorbital plexus into Eppendorf tubes containing ethylenediaminetetraacetic acid. The blood samples were collected at the following time points: 0 (predose), $0.5,1$, $2,3,4,6,8,12$ and 24 hours. The blood samples were then centrifuged at 5,000 rpm for 15 minutes. The plasma (supernatant) was collected in Eppendorf tubes and was precipitated using the procedure stated earlier, and the supernatant was stored in a freezer at $-20^{\circ} \mathrm{C}$, which was analyzed using high-performance liquid chromatography, as described earlier.

\section{Statistical analysis}

Statistical analysis was conducted using Statistica11 (StatSoft, Inc., now a part of Dell Software, Santa Clara, CA, USA). Results were expressed as mean \pm standard deviation. Comparison of marketed preparation, drug solution and formulation suspension data was done by analysis of 
variance (ANOVA), and $P$-value less than 0.05 was considered significant.

\section{Results and discussion}

DCNF was prepared from CNF by the physical adsorption method. During the preparation and optimization of DCNF, it was observed that after going through the process of freezedrying, DCNF was converted into a foam-like structure, ie, an aerogel. It was also observed that some physical changes took place after freeze-drying. Therefore, in vitro characterization was performed on both CNF and DCNF aerogels.

\section{Optimization of formulation}

To identify the optimum levels of different process variables influencing the response, ie, drug loading of bendamustine hydrochloride, two-factor central composite design, as suggested by the Design-Expert (Stat-Ease, Inc) software for DOE for the one dependent attribute (drug loading) was used. The individual and interactive effects of these process variables were studied by conducting the process at different levels of all the factors. The results of the experimental data and simulated values are listed in Table 1. According to the software-generated data, 13 formulations were prepared with $\mathrm{n}=3$.

\section{Effect of different factors on drug loading}

According to the 3D plot (Figure 1), it is evident that the drug loading increases with increase in the concentration of fibers, reaches a maximum and then becomes constant. Similarly, the analysis of effect of stirring time showed increase in the drug loading with increase in the stirring time up to a point, after which it remains almost constant.

Table I Drug loading with two-factor central composite design

\begin{tabular}{llll}
\hline $\begin{array}{l}\text { Serial } \\
\text { no }\end{array}$ & CNF ratio & $\begin{array}{l}\text { Stirring time } \\
\text { (hours) }\end{array}$ & $\begin{array}{l}\text { Drug } \\
\text { loading (\%) }\end{array}$ \\
\hline 1 & 3.0 & 8.0 & $18.98 \pm 1.57$ \\
2 & 1.8 & 5.5 & $11.46 \pm 2.63$ \\
3 & 1.8 & 5.5 & $14.51 \pm 1.89$ \\
4 & 1.8 & 9.04 & $14.35 \pm 3.21$ \\
5 & 3.5 & 5.5 & $20.16 \pm 2.64$ \\
6 & 1.8 & 5.5 & $13.33 \pm 2.11$ \\
7 & 0.1 & 5.5 & $0.22 \pm 1.21$ \\
8 & 1.8 & 5.5 & $12.62 \pm 1.66$ \\
9 & 0.6 & 3.0 & $0.46 \pm 0.32$ \\
10 & 3.0 & 3.0 & $6.08 \pm 0.86$ \\
11 & 1.8 & 1.96 & $3.12 \pm 1.2$ \\
12 & 1.8 & 5.5 & $13.24 \pm 1.43$ \\
13 & 0.6 & 8.0 & $0.81 \pm 0.43$ \\
\hline
\end{tabular}

Abbreviation: $\mathrm{CNF}$, cellulose nanofiber.

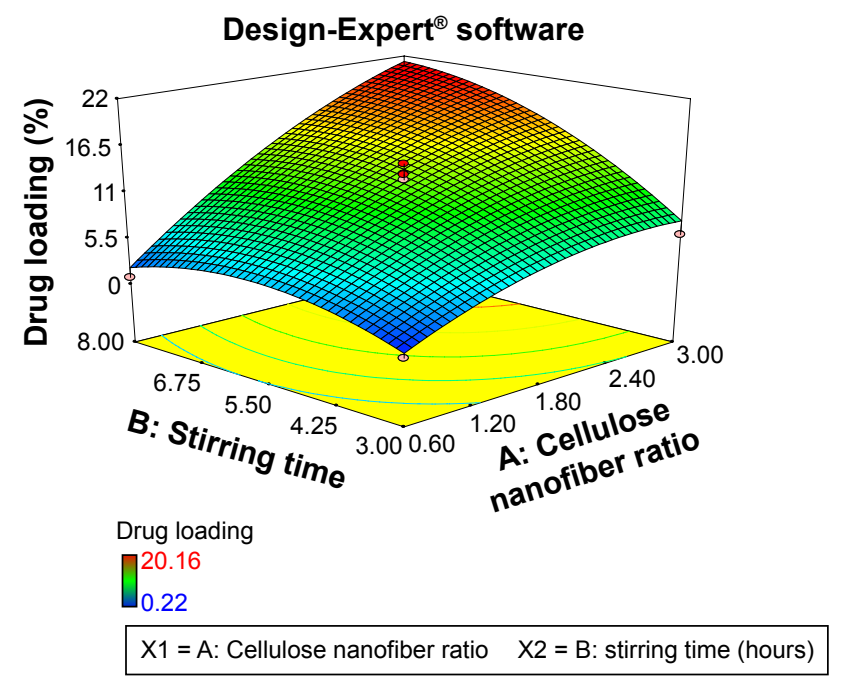

Figure I 3D response surface plot showing the influence of the polymer ratio and stirring time on drug loading.

The drug loading predicted by Design-Expert software was $21.3001 \%$, whereas the obtained value was $18.98 \% \pm 1.57 \%$, with entrapment efficiency of $77.6 \% \pm 1.58 \%$.

\section{Morphology determination by SEM}

For determining the surface characteristics of the formed $\mathrm{CNF}$ aerogel, SEM was used. There was a difference in the surface attributes of the CNF aerogel and the DCNF aerogel. The plain CNF aerogel (Figure 2A) showed a porous matrix, while in the DCNF aerogel, pores are much filled with matrixtype layered structure due to physical adsorption of the drug on the fibers (Figure 2B).

\section{Morphology determination by TEM}

The TEM image (Figure 3B) shows the loading of the drug molecules onto the nanofiber by the increase in diameter. The diameter of plain CNF was found to be $7.47 \pm 0.82 \mathrm{~nm}$ (Figure 3A), while in the case of DCNF, it was recorded to be $39.33 \pm 0.56 \mathrm{~nm}$ (Figure 3B). Several nodes are seen on the nanofiber, indicating the attachment of drug molecules.

\section{Swelling index in media with different $\mathrm{pH}$ values}

The swelling property of CNF aerogel and DCNF aerogel was studied in media with different $\mathrm{pH}$ values (1.2 and 7.4) for different time periods (12 and 24 hours). Difference in swelling tendency was observed, and the results are shown in Figure 4. Maximum swelling was seen at 24 hours, with swelling indexes of $254 \% \pm 19 \%$ and $225 \% \pm 15 \%$ for the $\mathrm{CNF}$ aerogel and DCNF aerogel, respectively, at $\mathrm{pH} 7.4$; and $210 \% \pm 18 \%$ and $189 \% \pm 12 \%$, respectively, at $\mathrm{pH} 1.2$. 

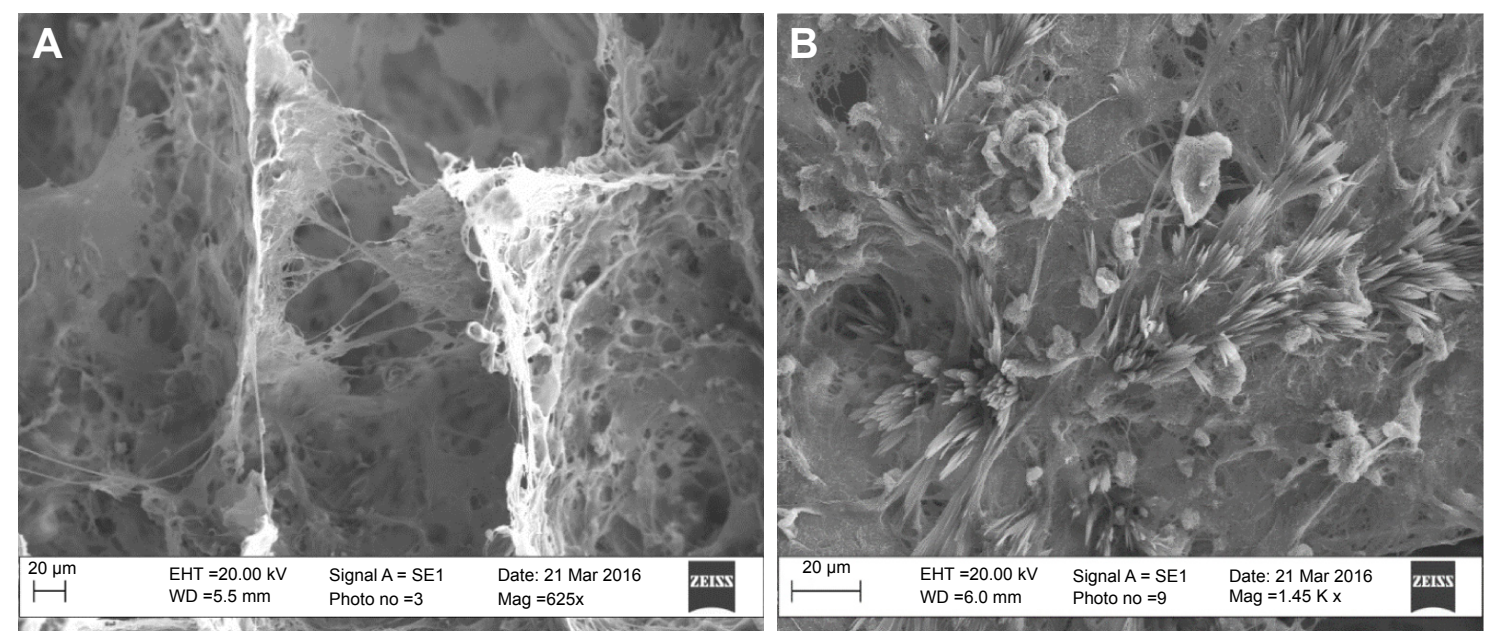

Figure 2 SEM images of (A) CNF aerogel and (B) DCNF aerogel.

Abbreviations: SEM, scanning electron microscopy; CNF, cellulose nanofiber; DCNF, drug-loaded CNF; WD, working distance; EHT, electron high tension.

High degree of swelling can be correlated to the water retained in the highly porous interconnected fibers; however, the difference in swelling behavior under different $\mathrm{pH}$ conditions is due to the characteristics of the cellulose. The findings are very well corroborated with findings reported by Silvestre et $\mathrm{al},{ }^{20}$ in which the authors reported that for $\mathrm{BC}$, the swelling index is less at $\mathrm{pH}$ values $<5$, then rises till $\mathrm{pH} 7$ and then again there is a slight decrease till $\mathrm{pH} 10$. The difference in the swelling indexes of the DCNF and CNF aerogels can be attributed to the decrease in number and accessibility of hydrophilic sites in the case of the DCNF aerogel due to occupancy by bendamustine.

\section{Mucoadhesive strength}

Figure 5A and $\mathrm{B}$ represents the mucoadhesive strength of the CNF and the DCNF aerogels. The mucoadhesive strength of

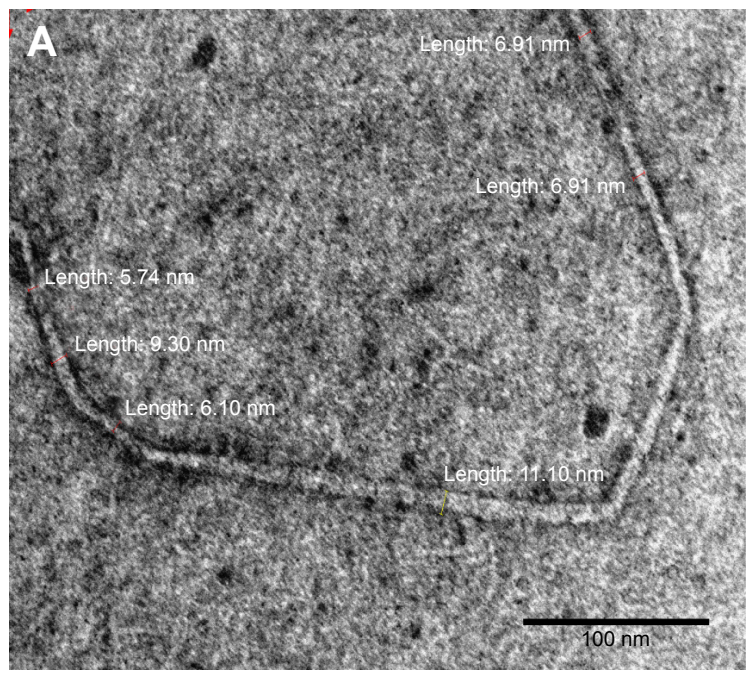

the blank nanofiber aerogel was found to be $36.9 \pm 3.8 \mathrm{~g} / \mathrm{cm}^{2}$, and that of the drug-loaded nanofiber aerogel was found to be $18.19 \pm 3.64 \mathrm{~g} / \mathrm{cm}^{2}$. The decrease in mucoadhesive strength in the case of DCNF aerogel can be attributed to the decrease in the availability of specific surface area of CNF for adhesion. ${ }^{17}$

\section{Tensile strength}

Tensile strength of the CNF formulation can be attributed to the fact that when water is removed from the cellulose fibers during drying, hydrogen bonds are formed between neighboring fibers, creating a tight fiber network. This process is irreversible and is known as "hornification", which imparts strength to CNF and makes it durable. ${ }^{12}$

Figure $6 \mathrm{~A}$ and $\mathrm{B}$ shows the tensile strength of $\mathrm{CNF}$ and DCNF aerogels. The tensile strength of CNF aerogel was found to be $122.69 \pm 17 \mathrm{~g} / \mathrm{cm}^{2}$, and that of the DCNF aerogel

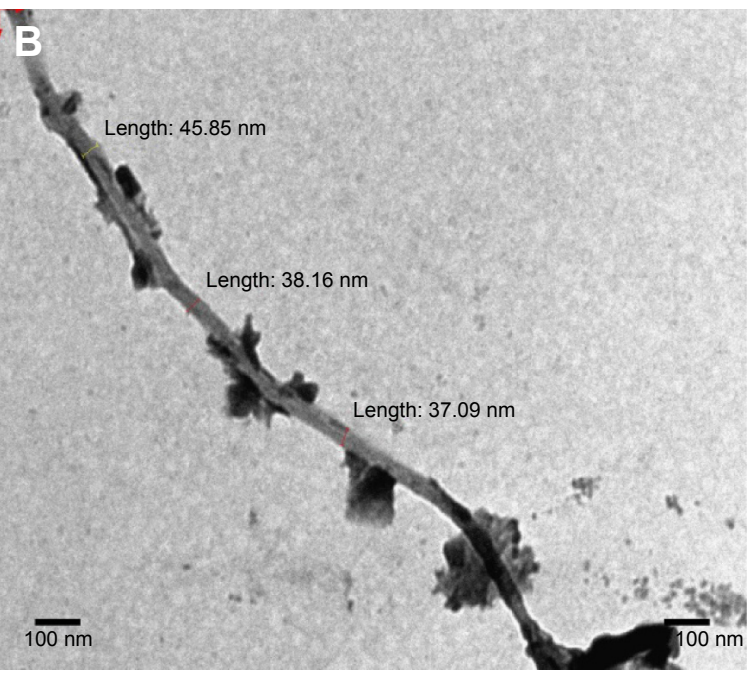

Figure 3 TEM images of (A) CNF and (B) DCNF aerogels.

Abbreviations: TEM, transmission electron microscopy; CNF, cellulose nanofiber; DCNF, drug-loaded CNF. 


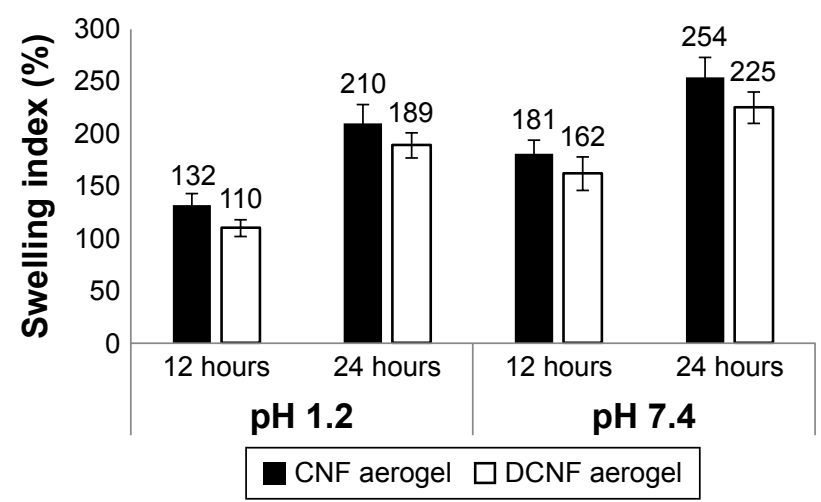

Figure 4 Swelling indexes of CNF and DCNF aerogels at different time intervals in media with different $\mathrm{pH}$ values. Error bar represents the standard deviation $(\mathrm{n}=6)$. Abbreviations: CNF, cellulose nanofiber; DCNF, drug-loaded CNF.

was found to be $115.3 \pm 11 \mathrm{~g} / \mathrm{cm}^{2}$. The low value of the tensile strength for DCNF aerogel may be attributed to the changes in the property of CNFs after drug loading, as the mechanical strength of fibers is inversely proportional to the diameter of the fibers. ${ }^{21}$

\section{Floating time}

The obtained DCNF aerogel floated for $\sim 450 \pm 30$ minutes in dissolution media of $\mathrm{pH} 7.4$ and $\mathrm{pH}$ 1.2. This property can be attributed to the extremely high surface area. Nanofibers with $100 \mathrm{~nm}$ diameter provide a specific area of $1,000 \mathrm{~m}^{2} / \mathrm{g}$. Increased surface area of the nanofibers contributes to the significant reduction of bulk density. ${ }^{17}$

\section{In vitro drug release study}

The in vitro drug release experiment was conducted for 24 hours using dialysis bags filled with pure drug and DCNF formulations. The result is depicted in Figure 7.
Approximately $88.12 \% \pm 3.33 \%$ of the pure drug was released in the first 6 hours from the drug solution, while only $53 \% \pm 2.79 \%$ of drug was released from the nanofiber formulation. The DCNF aerogel showed controlled release behavior, with $69.205 \% \pm 2.5 \%$ of the drug being released in 24 hours, while almost-complete drug release was seen within 12 hours from the drug solution. The dissolution behavior from the cellulose matrix is primarily dependent on the penetration of medium into the matrix and the swelling properties of the porous matrix formed after lyophilization, ${ }^{12}$ in addition to the dissolution of the drug from the matrix.

\section{Study of release kinetics}

The data obtained from the release study at $\mathrm{pH} 1.2$ was fitted into various release kinetics models - zero order, first order, Higuchi and Korsmeyer-Peppas models (Figure 8A-D).

The release data best fitted into the Korsmeyer-Peppas model (graph of log cumulative percentage of drug released versus $\log$ time); the exponent $n$ was calculated through the slope of the straight line. Generally, one of the two conditions exists. In Case 1, where the drug transport mechanism from spherical matrices is by Fickian diffusion, then $n \leq 0.43$ and if $0.435<\mathrm{n}<0.85$, it is anomalous (non-Fickian) transport; and in Case II, for values of $n \geq 0.85$, zero-order release kinetics is indicated. Model fitting revealed that the nanofiber formulation showed anomalous transport (non-Fickian) mechanism for the release of drug, where the value of $R^{2}$ was found to be 0.9736 and $n=0.613$. However, it does not always confirm that the CNF aerogel follows the Korsmeyer-Peppas model. Literature mentions that the release from the CNFs depends on the thickness of the film/aerogel ${ }^{22}$ and also on the property of the drug and the type of cellulose used in the formulation. ${ }^{12}$

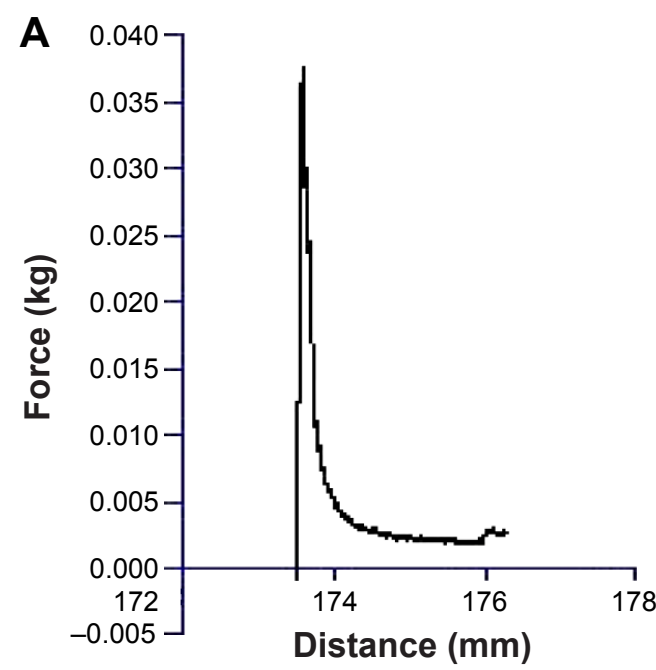

Figure 5 Mucoadhesive strength of (A) CNF aerogel and (B) DCNF aerogel. Abbreviations: CNF, cellulose nanofiber; DCNF, drug-loaded CNF.

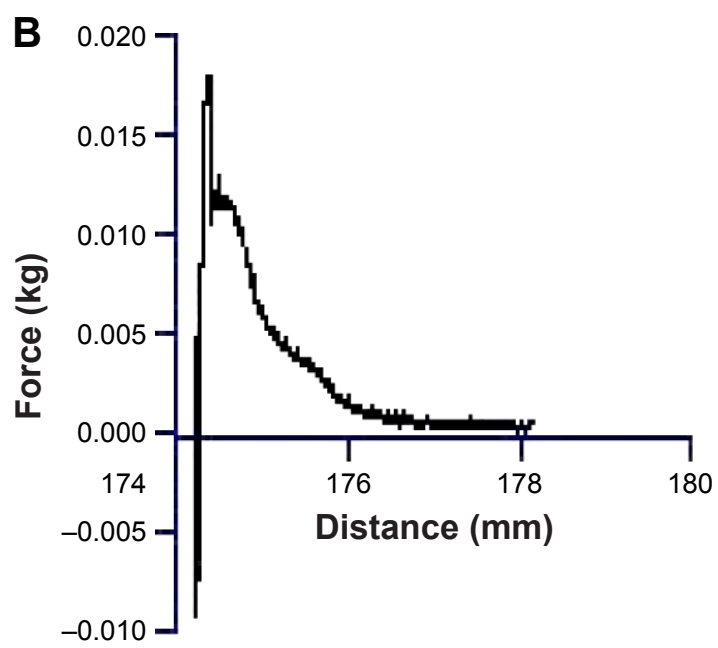




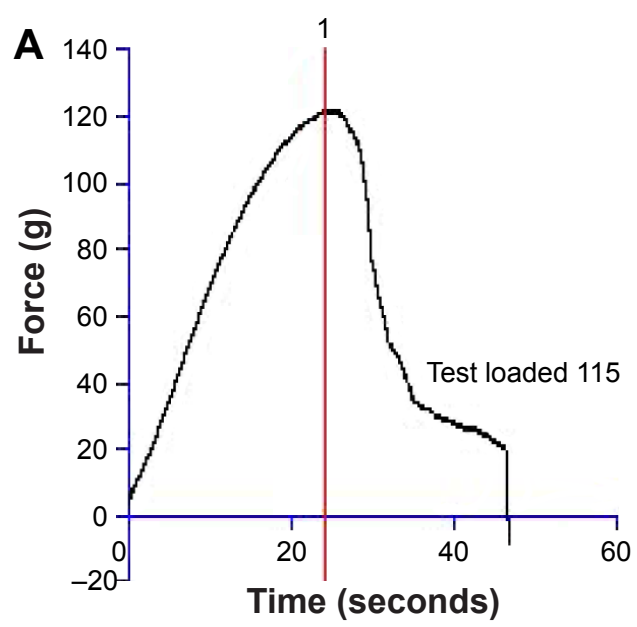

Figure 6 Tensile strength of (A) CNF aerogel and (B) DCNF aerogel. Abbreviations: CNF, cellulose nanofiber; DCNF, drug-loaded CNF.

\section{Comparison of drug release at different $\mathrm{pH}$ values}

The cumulative percentage release was determined in media of $\mathrm{pH} 1.2$ and $\mathrm{pH}$ 7.4. The release study conducted for 24 hours showed a difference in percentage release, which is depicted in Figure 7. Approximately $69 \% \pm 2.5 \%$ of drug was released in the medium of $\mathrm{pH} 1.2$, whereas $\sim 78 \% \pm 2.28 \%$ of drug was released in the medium of $\mathrm{pH}$ 7.4. The difference in drug release in the two media with different $\mathrm{pH}$ values can be attributed to the difference in swelling behavior of the CNF aerogel in the two media.

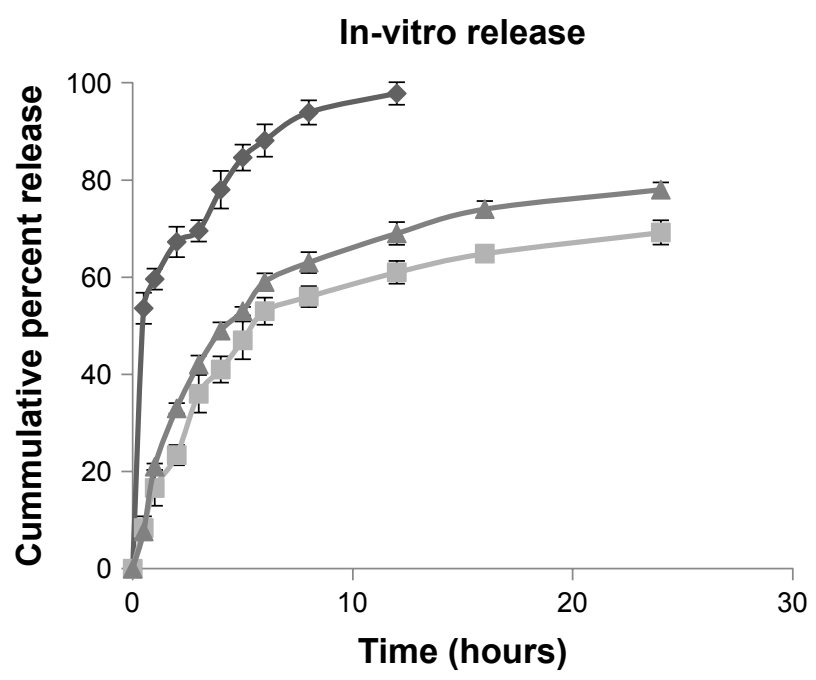

- Cumulative \% release from drug solution at $\mathrm{pH} 1.2$
- Cumulative \% release from DCNF at $\mathrm{pH} 1.2$
- Cumulative \% release from DCNF at $\mathrm{pH} 7.4$

Figure $7 \mathrm{In}$ vitro drug release in media with $\mathrm{pH} \mathrm{I.2}$ and $\mathrm{pH} 7.4$ at different time intervals.

Abbreviation: DCNF, drug-loaded cellulose nanofiber.

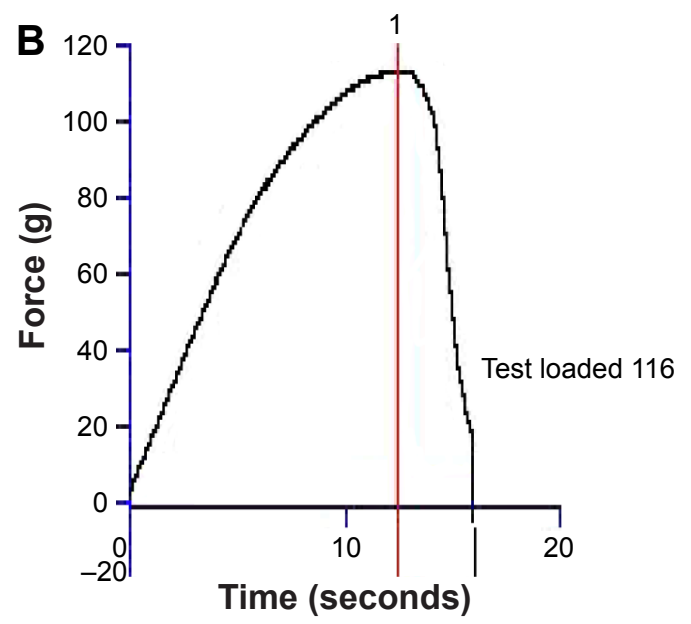

A significant increase in release was observed at $\mathrm{pH} 7.4$ due to the higher swelling index, which ultimately leads to higher drug loading.

\section{In vivo study \\ HPLC analysis}

The HPLC method reported by Xie et a ${ }^{18}$ was used, with slight modification, to determine the drug concentration in plasma. Figure 9 represents the HPLC peak of the drug.

\section{Pharmacokinetic study}

The pharmacokinetic studies were performed to evaluate and compare the kinetics as well as the blood levels of drug and the NF formulation. Blood samples were collected at various time points, processed and analyzed by HPLC. The retention time was 13.842 minutes and $R^{2}$ was 0.9961 . The concentration of drug at different time intervals for different groups of rats is demonstrated in Figure 10 and the pharmacokinetic parameters are given in Table 2 .

As shown in Table 2, the maximum plasma concentration $\left(C_{\max }\right)$ was found to be $2,700 \pm 67 \mathrm{ng} / \mathrm{mL}$ for the marketed drug preparation administered intravenously as well as $1,750 \pm 43$ and $1,563 \pm 58 \mathrm{ng} / \mathrm{mL}$, respectively, for the pure drug and drug-loaded formulation when given orally. The time taken to reach $C_{\max }\left(T_{\max }\right)$ for the marketed drug preparation administered intravenously was found to be 30 minutes, while for the pure drug solution and DCNF formulation given orally, it was found to be 1 and 4 hours, respectively. The area under the curve (AUC) was found to be $2,842.5$, $4,951.5$ and $16,089.75 \mathrm{ng} \cdot \mathrm{h} / \mathrm{mL}$ for the marketed formulation administered intravenously, drug solution given orally and DCNF given orally. 

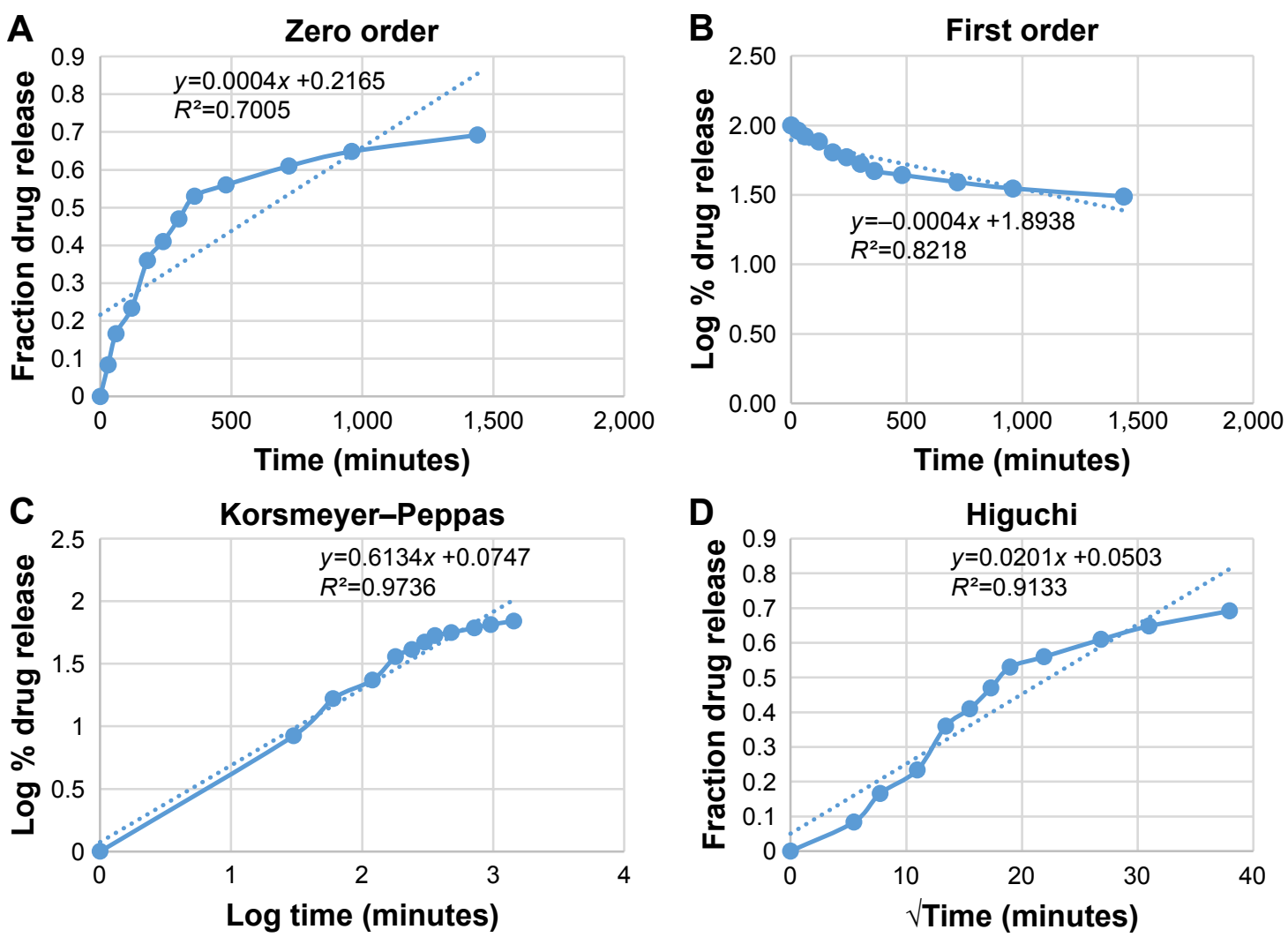

Figure 8 Study profiles of release kinetics.

Notes: (A) Zero order; (B) first order; (C) Korsmeyer-Peppas; (D) Higuchi.

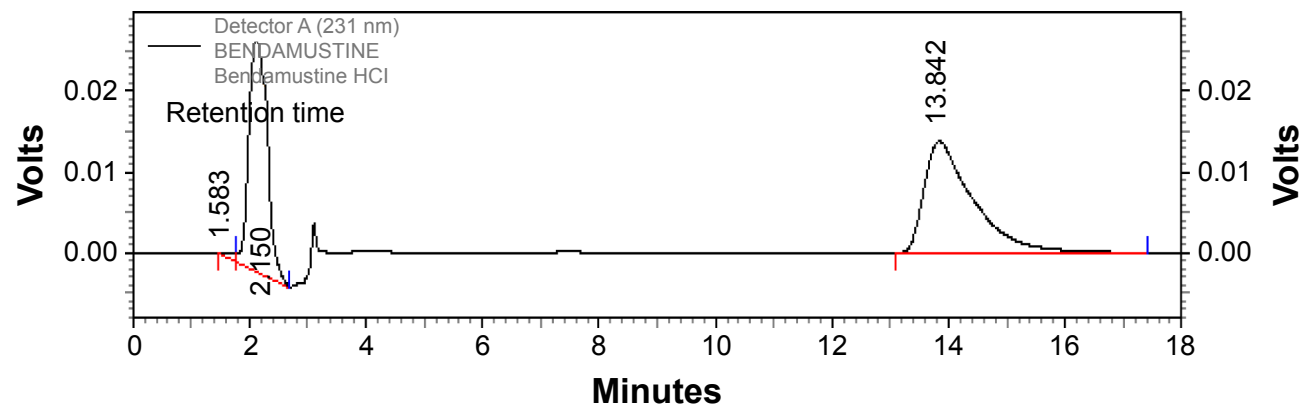

Figure 9 HPLC chromatogram of bendamustine hydrochloride in rat plasma.

Abbreviation: HPLC, high-performance liquid chromatography.

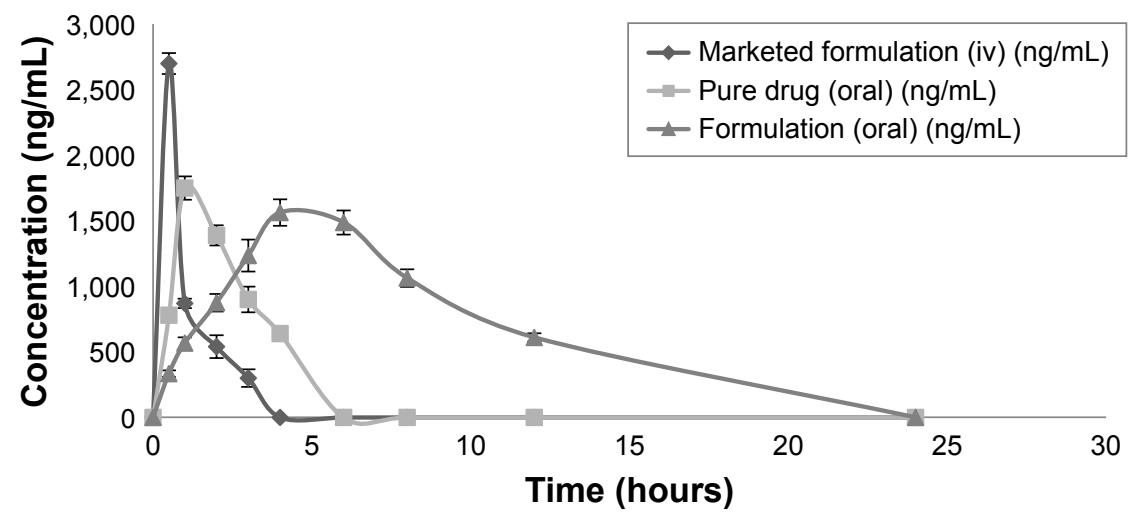

Figure 10 Drug concentrations in blood vs time. Error bar represents standard deviation $(n=6)$. Abbreviation: iv, intravenous. 
Table 2 Pharmacokinetic parameter results

\begin{tabular}{|c|c|c|c|c|c|}
\hline Samples & $C_{\max }, \mathrm{ng} / \mathrm{mL}$ & $\begin{array}{l}T_{\text {max }}, \\
\text { minutes }\end{array}$ & $\begin{array}{l}\text { Relative } \\
\text { bioavailability }\end{array}$ & $\begin{array}{l}\text { Absolute } \\
\text { bioavailability }\end{array}$ & $\begin{array}{l}\text { AUC, } \\
\mathrm{ng} \cdot \mathrm{h} / \mathrm{mL}\end{array}$ \\
\hline Marketed preparation (iv) & $2,700 \pm 67$ & 30 & - & - & $2,842.5$ \\
\hline Drug solution (oral) & $\mathrm{I}, 750 \pm 43$ & 60 & - & 1.74 & $4,951.5$ \\
\hline Formulation suspension (oral) & 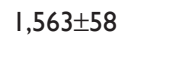 & 240 & $\begin{array}{l}3.25 \text { wrt oral } \\
\text { drug solution }\end{array}$ & 5.66 & $16,089.75$ \\
\hline
\end{tabular}

Notes: $C_{\max }$, maximum plasma concentration; $T_{\max }$, time required to reach $C_{\max }$.

Abbreviations: AUC, area under the curve; iv, intravenous; wrt, with respect to.

The absolute bioavailability and relative bioavailability of the formulation were found to be increased by 5.66 and 3.25 times, respectively, compared to the intravenous and oral bioavailability of the pure drug solution. Hence, it is evident that the formulation is able to enhance the oral bioavailability of the drug by providing controlled drug release.

\section{Conclusion}

During our investigations on CNF aerogels as a drug delivery system, we observed the difference in physical and mechanical properties of aerogels due to drug loading. The major characteristics of a delivery system, such as floating time, mucoadhesive properties and swelling index, were affected by drug loading. CNF aerogels are a unique and promising natural material with huge pharmaceutical potential in biomedicine as drug carrier. Because of CNF's extremely high surface area, hygroscopic nature and swelling tendency, it allows maximum drug loading by physical adsorption. Moreover, its swelling ability allows the drug to be released in a controlled manner from the CNF aerogel. Moreover, the floating tendency and the mucoadhesive property of the $\mathrm{CNF}$ aerogel present it as a successful gastroretentive drug delivery system. From the results of the study, it can be concluded that the CNFs, due to their characteristic features, are suitable for the loading of water-soluble drugs by physical adsorption. Because the aerogels possess good tensile strength due to the hydrogen bonding between the molecules, they are more suitable as transdermal drug delivery systems. Not only tensile strength, their drug release pattern and hygroscopic nature show suitability for drug delivery through skin. Thus, they generate a huge possibility for implants and transdermal drug delivery systems. The floating tendency is due to the decrease in bulk density of the NFs due to entrapment of air within. Though the CNFs floated only for 7 hours and 30 minutes, modification of cellulose properties, such as making it hydrophobic, might help it to float for longer duration of time if it is supposed to be used for oral drug delivery.

\section{Acknowledgments}

The authors are thankful to Forest Products Laboratory (University of Maine, Orono, ME, USA) for providing the nanocellulose fibers used in this research work. We are also grateful to Fresenius Kabi Oncology (Gurgaon, Haryana, India), for providing a gift sample of bendamustine hydrochloride. Dr Sushama Talegaonkar is also thankful to University Grants Commission, Government of India, for the financial support.

\section{Disclosure}

The authors report no conflicts of interest in this work.

\section{References}

1. Deshpande AA, Rhodes CT, Shah NH, Malick AW. Controlled-release drug delivery systems for prolonged gastric residence: an overview. Drug Dev Ind Pharm. 1996;22(6):531-539.

2. Silvia M, Kawthar B, Gilles P. Oral delivery of anticancer drugs I: general considerations. Drug Discov Today. 2012;18(1-2):25-34.

3. Murphy CS, Pillay V, Choonara YE, Toit LC. Gastroretentive drug delivery systems: current developments in novel system design and evaluation. Curr Drug Deliv. 2009;6(5):451-460.

4. Abe K, Iwamoto S, Yano H. Obtaining cellulose nanofibers with a uniform width of $15 \mathrm{~nm}$ from wood. Biomacromolecules. 2007;8(10): 3276-3278.

5. Zhang Y, Lim CT, Ramakrishna S, Huang ZM. Recent development of polymer nanofibers for biomedical and biotechnological applications. J Mater Sci Mater Med. 2005;16(10):933-946.

6. Doshi J, Reneker DH. Electrospinning process and applications of electrospun fibers. J Electrostat. 1995;35(2):151-160.

7. Valo H, Kovalainen M, Laaksonen P, et al. Immobilization of proteincoated drug nanoparticles in nanofibrillar cellulose matrices - enhanced stability and release. J Control Release. 2011;156(3):390-397.

8. Frone AN, Panaitescu DM, Donescu D. Some aspects concerning the isolation of cellulose micro- and nano-fiber. UPB Sci Bull. 2011; 73(2):133-152.

9. Jung JY, Khan T, Park JK, Chang HN. Production of bacterial cellulose by Gluconacetobacter hansenii using a novel bioreactor equipped with a spin filter. Korean J Chem Eng. 2007;24(2):265-271.

10. Czaja WK, Young DJ, Kawecki M, Brown M. The future prospects of microbial cellulose in biomedical applications. Biomacromolecules. 2007;8(1):1-12.

11. Kolakovic R, Peltonen L, Laukkanen A, Hirvonen J, Laaksonen T. Nanofibrillar cellulose films for controlled drug delivery. Eur J Pharm Biopharm. 2012;82(2):308-315.

12. Valo H, Arola S, Laaksonen $\mathrm{P}$, et al. Drug release from nanoparticles embedded in four different nanofibrillar cellulose aerogels. Eur J Pharm Sci. 2013;50(1):69-77. 
13. Sharma H, Kumar K, Choudhary C, Mishra PK, Vaidya B. Development and characterization of metal oxide nanoparticles for the delivery of anticancer drug. Artif Cells Nanomed Biotechnol. 2016;44(2):672-679.

14. Meng ZX, Xu X, Zheng W, et al. Preparation and characterization of electrospun PLGA/gelatin nanofibers as a potential drug delivery system. Colloids Surf B Biointerfaces. 2011;84(1):97-102.

15. Thirawong N, Nunthanid J, Puttipipatkhachorn S, Sriamornsak P. Mucoadhesive properties of various pectins on gastrointestinal mucosa: an in vitro evaluation using texture analyzer. Eur J Pharm Biopharm 2007;67(1):132-140.

16. Singh H, Sharma R, Joshi M, Garg T, Goyal AK, Rath G. Transmucosal delivery of docetaxel by mucoadhesive polymeric nanofibers. Artif Cells Nanomed Biotechnol. 2015;43(4):263-269.

17. Malik R, Garg T, Goyal AK, Rath G. Diacerein-loaded novel gastroretentive nanofiber system using PLLA: development and in vitro characterization. Artif Cells Nanomed Biotechnol. 2016;44(3): 928-936.
18. Xie F, Cheng Z, Cheng H, Yu P. Simultaneous determination of bendamustine and its active metabolite, gamma-hydroxy-bendamustine in human plasma and urine using HPLC-fluorescence detector: application to a pharmacokinetic study in Chinese cancer patients. J Chromatogr B Analyt Technol Biomed Life Sci. 2014;960:98-104.

19. Colledge J, inventor. Oral Dosage Forms of Bendamustine; Astellas Deutschland Gmbh, assignee. US 20140018334 A1. 2014, Jan 16.

20. Silvestre AJ, Freire CS, Neto CP. Do bacterial cellulose membranes have potential in drug-delivery systems? Expert Opin Drug Deliv. 2014;11(7):1113-1124.

21. Chew SY, Hufnagel TC, Lim CT, Leong KW. Mechanical properties of single electrospun drug-encapsulated nanofibres. Nanotechnolgy. 2006;17(5):3880-3891.

22. Haimer E, Wendland M, Schlufter K, et al. Loading of bacterial cellulose aerogels with bioactive compounds by antisolvent precipitation with supercritical carbon dioxide. Macromol Symp. 2010;294(2):64-74.
International Journal of Nanomedicine

\section{Publish your work in this journal}

The International Journal of Nanomedicine is an international, peerreviewed journal focusing on the application of nanotechnology in diagnostics, therapeutics, and drug delivery systems throughout the biomedical field. This journal is indexed on PubMed Central, MedLine, CAS, SciSearch $®$, Current Contents $\AA /$ Clinical Medicine,

\section{Dovepress}

Journal Citation Reports/Science Edition, EMBase, Scopus and the Elsevier Bibliographic databases. The manuscript management system is completely online and includes a very quick and fair peer-review system, which is all easy to use. Visit http://www.dovepress.com/ testimonials.php to read real quotes from published authors.

Submit your manuscript here: http://www.dovepress.com/international-journal-of-nanomedicine-journal 